\title{
EchoGéo
}

29 | 2014

The Political Ecology of Conservation and

Development Territories

\section{Territorialisation et pouvoir : la Political Ecology des territoires de conservation et de développement}

Introduction

Thomas J. Bassett and Denis Gautier

\section{(2) OpenEdition}

\section{Journals}

\section{Electronic version}

URL: https://journals.openedition.org/echogeo/14044

DOI: 10.4000/echogeo.14044

ISSN: 1963-1197

\section{Publisher}

Pôle de recherche pour l'organisation et la diffusion de l'information géographique (CNRS UMR 8586)

\section{Electronic reference}

Thomas J. Bassett and Denis Gautier, "Territorialisation et pouvoir : la Political Ecology des territoires de conservation et de développement", EchoGéo [Online], 29 | 2014, Online since 18 November 2014, connection on 31 July 2021. URL: http://journals.openedition.org/echogeo/14044 ; DOI: https:// doi.org/10.4000/echogeo.14044

This text was automatically generated on 31 July 2021.

EchoGéo est mis à disposition selon les termes de la licence Creative Commons Attribution - Pas d'Utilisation Commerciale - Pas de Modification 4.0 International (CC BY-NC-ND) 


\section{Territorialisation et pouvoir : la Political Ecology des territoires de conservation et de développement}

Introduction

Thomas J. Bassett and Denis Gautier

\section{Introduction}

$1 \mathrm{Au}$ nom de la conservation environnementale, de la sécurité alimentaire et du développement durable, un groupe diversifié d'acteurs public et privés s'affairent à diviser les Suds en territoires de conservation et de développement à différentes échelles. Plusieurs objectifs et processus conduisent ces stratégies spatiales à reconfigurer l'accès aux ressources, leur contrôle et leur gestion. La production de territoire pour contrôler les populations et les ressources est une pratique ancienne (Elden, 2013 ; Sack, 1986 ; Scott, 1998). Les parcs nationaux, réserves de biosphère et zones d'exportation sont des exemples bien connus des pratiques territoriales étatiques dans la perspective de gouverner la conservation et le développement. Les projets territoriaux actuels s'appuient en les adaptant sur ces modèles et pratiques bien connus (Neumann, 2004 ; Wilshusen et al., 2002). Ce qui est nouveau dans la période actuelle est la diversification des acteurs, des institutions et des pratiques spatiales impliquées dans la territorialisation des terres et des eaux de la planète (Fairhead et al., 2012). Les partenariats publique-privé créent des couloirs de croissance agricole en Tanzanie et au Mozambique. Les gouvernements en Amérique du Sud et en Asie du Sud-Est travaillent avec des organisations internationales, des ONG environnementalistes et des institutions financières pour découper les forêts tropicales en concessions destinées à séquestrer du carbone en lien avec des systèmes de compensation de carbone et à conserver la biodiversité. En Afrique de l'Ouest, les gouvernements octroient des concessions en milieu rural à des entreprises d'agrobusiness qui détiennent le monopole du marché des intrants et des produits agricoles. Et un peu partout, les 
approches territoriales telles que la gestion de terroir et les technologies géo-spatiales sont utilisées pour la privatisation des espaces communs (Bassett, Blanc-Pamard, Boutrais, 2007). Ces pratiques spatiales et interventions nouvelles s'ajoutent à une longue liste de territoires de conservation et de développement préexistants: zones tampons et corridor de biodiversité dans les programmes intégrés de conservation et de développement, parcs internationaux de la Paix et réserves transfrontalières, accords d'échanges dette contre nature, et réserves extractives. Le marché de la protection de l'environnement, les discours politiques scientifiques, et l'accaparement des terres sont les principaux éléments moteurs de ces nouvelles enclosures (Fairhead et al., 2012).

2 Le concept de "territoire» et les termes associés de "territorialités » et de «territorialisation» sont relativement peu étudiés par les géographes humains et sociaux (Elden, 2010). Il est de ce fait important de définir ces termes dans la perspective d'éclairer l'approche political ecology des territoires de conservation et de développement qui constitue le thème de ce numéro spécial d'échoGéo. Le territoire est compris ici comme un espace socialement construit qui est caractérisé par ses origines historiques, culturelles, techniques, et politico-économiques (Elden, 2010). Les territoires peuvent être caractérisés comme des «espaces autonomes, distinctifs, délimitées, mesurables et transmissibles, qui sont créés délibérément dans la perspective d'atteindre certains objectifs sociaux» (Murphy, 2012, p. 164). Le territoire est à la fois une relation et un résultat du processus de territorialité que Robert Sack définit comme « une tentative par un individu ou un groupe d'affecter, d'influencer ou de contrôler les personnes, les phénomènes et les relations en délimitant et en revendiquant le contrôle sur un espace géographique » (Sack, 1986, p. 19). En tant que stratégie, la territorialité est inextricablement liée aux relations sociales qui structurent les aspirations et les possibilités de produire des territoires. Cette approche relationnelle de la territorialité, combinée à la conceptualisation de Sack, enrichit la notion de territorialité qui peut être alors comprise comme une stratégie qui produit des relations sociales dans le même temps où elle est produite par elles (Murphy, 2012, p. 169).

3 Le concept de territorialisation renvoie à des projets territoriaux spécifiques au sein desquels divers acteurs déploient des stratégies territoriales (territorialité) pour produire des espaces délimités et contrôlés (territoire) pour atteindre certains buts. Un objectif commun de la territorialisation est de gouverner les personnes et les ressources localisées et autour du territoire (Scott, 1998). Le contrôle peut être autoritaire comme dans le cas du déguerpissement de populations de leurs terres ancestrales. Cependant, la "conservation coercitive " n'est qu'une des nombreuses modalités de la territorialisation (Peluso, 1993). Celle-ci peut aussi s'exprimer indirectement à travers la prescription ou la proscription de certaines activités qui affectent l'accès aux ressources, leur contrôle et leur gestion. Cette reconfiguration socio-spatiale est typiquement finalisée par l'établissement de nouvelles lois, normes et autorités qui modifient le rapport humain-environnement. Le processus est aussi caractérisé par le déploiement de discours sur l'environnement et le développement. Ces discours présentent souvent les acteurs étatiques comme des gestionnaires éclairées des ressources naturelles qui sont capable de résoudre les problèmes environnementaux et de développement d'une manière responsable et durable. Inversement, les usagers des ressources sont généralement décrits par les acteurs 
étatiques comme des acteurs destructeurs, inefficaces et ignorants qui ont besoin d'être réorganisés et disciplinés à travers des arrangements socio-spatiaux conçus par l'État. De nouvelles subjectivités et collaborations mais aussi formes de résistance peuvent émerger au sein de ces géographies de l'environnement et du développement remodelées.

4 Ce numéro spécial d'échoGéo contribue à la littérature sur le concept de territorialisation en engageant un débat critique sur la notion de "contrôle par la territorialisation» qui est une composante théorique fondamentale de ce corpus de littérature. Les auteurs de ce numéro spécial dépassent la notion d'une territorialisation relevant de l'État et fonctionnaliste, dans laquelle les pauvres sont invariablement dépossédés de l'accès aux ressources, leur contrôle et leur gestion au profit d'acteurs plus puissants dans le processus de territorialisation. Ils réalisent cet apport théorique en analysant la production de territoires de conservation et de développement au travers du prisme de la political ecology. La Political ecology est une approche interdisciplinaire pour étudier l'accès aux ressources naturelles, leur contrôle et leur gestion qui fait ressortir l'importance réciproque des multiples acteurs (humain et non humain) à différentes échelles dans la durée avec une attention particulière pour l'influence des relations politiques et biophysiques des dynamiques naturesociété. Les perspectives de la Political ecology s'appuient sur une diversité de théories écologiques et sociales pour analyser les processus qui façonnent les relations humainenvironnement. Dans des contextes urbains et ruraux ainsi que terrestres et marins variés, les cinq articles prennent une approche relationnelle de leurs cas d'étude qui démontre les origines polycentriques et la contestation des limites des territoires de conservation et de développement. Les cas d'étude et les cadres d'analyse des auteurs de ce numéro spécial illustrent ce que nous nommerons ici une political ecology de la territorialisation. La suite de cette introduction met en évidence les principaux éléments de cette approche innovante de la territorialisation avec une emphase sur la pertinence théorique des résultats des auteurs pour la notion clé de contrôle par la territorialisation.

\section{La territorialisation en tant que processus polycentrique}

5 En décalage par rapport aux travaux dominants de la littérature sur la territorialisation dont l'État est l'acteur principal, les cas d'étude rassemblés dans ce numéro illustrent bien que la production de territoires provient de multiples sources et localisations. Dans ses réflexions théoriques sur la territorialisation, Fletcher Chamara-Huff démontre comment les tentatives de l'État pour territorialiser les espaces maritimes peut résulter dans des territorialités instables et multiples. Qu'il s'agisse d'un territoire étatique d'accès libre ou d'un territoire communautaire, le processus de territorialisation étatique peut conduire à un contrôle territorial strict ou à une reterritorialisation par l'État ou par des populations locales. Fletcher Chamara-Huff considère la territorialisation comme un mouvement politique qui produit de nouvelles pratiques spatiales ainsi que de nouvelles représentations de l'espace. Il soutient que les relations de pouvoir entre l'État, les océanologues, les sociologues et la société civile autour de la création des aires de protections marines conduit à une grande diversité de processus de territorialisation. 
6 Dans son analyse des discours sur les politiques de conservation au Bénin, Fanny Pochet montre comment des ONG environnementales internationales sont devenues des acteurs clés dans la mise en place de territoires de conservation. Alors que le discours conservationniste dominant entre 1992 et 2002 promouvait la participation des communautés riveraines des aires protégées dans les programmes de conservation, les ONG internationales se sont livrées à un plaidoyer pour un retour à un modèle de protection de la nature excluant l'humain ("back to the barriers"). Ce discours a conduit à une extension sans précédent des aires protégées qui couvrent désormais $23 \%$ du territoire national du Bénin.

7 Nicole List montre comment les paysans de Pikine dans la banlieue de Dakar, sans sécurité foncière, ont adopté une stratégie territoriale et construit des "alliances territoriales" pour maintenir le contrôle sur leurs exploitations urbaines que les entreprises agro-industrielles et des compagnies immobilières cherchaient à convertir en d'autres usages. Les agriculteurs urbains de Pikine Nord ont créé un territoire de conservation et de développement de petite dimension en rachetant des terres agricoles, grâce à la création d'une association d'agriculteurs puissante et des alliances avec certains cadres de l'administration locale et centrale. Le succès de cette « alliance territoriale» pour contrer les entreprises agro-industrielles et les compagnies immobilières dans leurs stratégies d'accaparement des terres agricoles démontre que les motivations pour créer du territoire peuvent venir de processus ascendants comme descendants.

8 Sarah Audouin et Alexis Gonin présentent un cas similaire de territorialisation ascendante dans le sud-ouest Burkinabè où les autorités coutumières organisent l'implantation des vergers d'anacardiers des agriculteurs migrants en des lieux spécifiques. Une agrégation spatiale des vergers est ainsi réalisée et crée un développement territorial attractif pour les commerçants d'anacarde asiatiques. Cette construction territoriale délibérée se réalise au détriment des éleveurs transhumants dont l'accès aux pâturages est réduit par l'expansion massive des vergers d'anacardiers dans le paysage.

\section{Les motivations multiples pour la territorialisation}

9 La perspective dominante que les États adoptent comme stratégie territoriale pour contrôler les personnes et les ressources réduit les multiples objectifs de l'État en un seul objectif. La contribution de Delphine Ayerbe démontre que les administrations locales et centrales sont désireuses de restreindre d'accès des terres reforestées en marge d'Addis Abeba en Éthiopie pour de multiples raisons. Très clairement, les municipalités et les sociétés parapubliques qui gèrent les plantations d'eucalyptus limitent l'accès des populations locales à ces forêts aménagées. Toutefois, l'État permet aux femmes riveraines de ramasser les branches tombées et le bois mort pour le vendre sur les marchés informels de cette capitale bourgeonnante. Ce droit d'accès contrôlé ne comble pas seulement les besoins en énergie domestiques mais sert également les objectifs de l'aménagement des forêts en réduisant les risques de feu et de maladie dans les plantations. Les gestionnaires forestiers vendent aussi du bois de service et d'œuvre aux charpentiers et aux entreprises de construction pour le développement de l'habitat et le marché du meuble. Delphine Ayerbe soutient que ce contrôle du marché du bois par l'État est efficace pour contrôler les prix du bois qui entre dans Addis depuis les 
provinces reculées afin de le rendre abordable pour les urbains. Les plantations sont également valorisées comme réserves de bois en vue des besoins de développement futurs comme pour la papeterie qui est planifiée. De surcroit, la localisation des plantations en périphérie de la ville est envisagée par l'État comme espace d'extension urbaine possible. Un objectif final de ce territoire de conservation/développement est de servir de modèle à la gestion des ressources naturelles. L'État se considère lui-même comme un gestionnaire avisé des ressources naturelles du pays et considère que ses pratiques devraient être adoptées par les agences gouvernementales éthiopiennes. Les objectifs multiples des plantations d'eucalyptus à Addis Abeba servent à élargir la perspective assez étroite de la territorialisation qui est de contrôler l'accès aux ressources, leur contrôle et leur gestion.

\section{Les politiques de territorialisation}

10 Les politiques de fabrique des territoires ont toujours été marquées par les conflits et la violence du fait que l'État se heurte aux revendications des populations préexistantes au territoire qui défendent leurs droits légitimes sur les ressources. Les cas d'étude rassemblés ici illustrent les enjeux pour l'accès aux ressources et leur contrôle et les luttes pour le pouvoir qui leur sont liées dans le processus de territorialisation. Fletcher Chamara-Huff discute de la stratégie politique des pêcheurs des îles Exumas dans les Bahamas qui ont initialement cherché à défendre les droits d'un accès libre aux lieux de pêche pour tous les citoyens menacés par la création d'une aire de protection marine. Toutefois, les pêcheurs ont changé de tactique en adoptant les discours conservationnistes et les pratiques territoriales de l'État en proposant une reterritorialisation de l'aire protégée pour y inclure la pêche de subsistance locale. Cette alternative à l'aire de protection marine en tant que territoire d'exclusion créerait un nouveau territoire avec un accès réservé aux pêcheurs locaux uniquement.

11 Dans le cas du Bénin, l'analyse des discours réalisée par Fanny Pochet révèle les changements dans les stratégies de conservation environnementale au cours de l'histoire. Son article démontre que les discours conservationnistes ont évolué de la "conservation-forteresse " de l'époque coloniale à la "conservation basée sur les communautés " à partir des années 1980 dans un contexte d'incapacité des États à contrôler efficacement les territoires de conservation. Puis plus récemment, un discours de «retour aux barrières" promu par les ONG environnementales internationales a remplacé l'approche impliquant la participation des communautés. En dépit de tous ces changements de discours, un thème a été récurrent dans toutes les politiques de conservation: la restriction de l'accès des populations aux ressources. Cette restriction va des évictions forcées et de la criminalisation des activités de gestion des ressources par les populations locales comme la chasse, à la décision de savoir qui participe à la construction des territoires de conservation. Les politiques et territoires de conservation ont invariablement eu un effet sur les conflits entre les usagers locaux des ressources naturelles, l'État, et les organisations environnementales internationales.

12 Afin prendre le contrôle des terres gérées par les agriculteurs urbains de Pikine pour y implanter un projet immobilier, les autorités gouvernementales locales et centrales et un promoteur immobilier ont envoyé un bulldozer et les forces de police pour exproprier de leurs terres les exploitants agricoles qui n'accédaient pas à leur demande 
de vendre leur exploitation. Ces mesures coercitives combinées à une stratégie de diviser pour mieux régner ont abouti à des expropriations foncières à Pikine Ouest. Cependant, cette stratégie a été mise en échec à Pikine Nord où les exploitants agricoles ont combattu la police et stoppé l'avancée du bulldozer. Nicole List soutient que la victoire des exploitants agricoles de Pikine Nord dans la défense de leurs terres contre le développement urbain est due à une forte " alliance territoriale " qui a rassemblé des organisations paysannes, des ONG locales et internationales, des fonctionnaires et des autorités traditionnelles. Elle défend l'idée que les alliances territoriales stratégiques multi-niveaux sont de plus en plus fréquentes dans le contexte des politiques de décentralisation. Ces alliances remplacent progressivement les formes de gouvernance urbaine où les autorités du gouvernement central exercent le pouvoir dominant. Dans ce contexte de modification des bases du pouvoir, d'urbanisation galopante et de spéculation foncière, Nicole List soutient que les politiques de territorialisation sont liées à la compétition entre alliances territoriales qui évoluent elles-mêmes en fonction des changements de contexte et d'enjeux.

\section{Revendications différenciées des ressources et territoires}

13 L'image d'un État monolithique concevant des schémas territoriaux aux dépens d'une population soumise indifférenciée est mise à mal par les alliances territoriales qui caractérisent les conflits pour les terres agricoles décrits par Nicole List dans la périphérie de Dakar, Sénégal ou par Sarah Audouin et Alexis Gonin au Burkina Faso. L'État est fragmenté en des services des gouvernements centraux, régionaux et locaux dont les membres participent à des alliances territoriales en compétition. Les élus adoptent des positions différentes de celles des bureaucrates gouvernementaux dans les organismes de gestion foncière qui agissent en fonction d'un ensemble différent d'incitations et de récompenses. Cette diversité institutionnelle au niveau gouvernemental est contrebalancée par une diversité d'acteurs et de logiques parmi les usagers des ressources à l'échelle du paysage. Certains agriculteurs urbains et leaders d'associations paysannes vendent la terre aux promoteurs immobiliers tandis que d'autres campent sur leurs positions et maintiennent le contrôle sur leurs terres agricoles.

14 Les différences entre les usagers des ressources, en particulier les inégalités des droits d'accès aux ressources, sont particulièrement importantes dans les espaces d'expansion des vergers d'anacardiers dans le Sud-Ouest du Burkina Faso. Les principaux acteurs de cette expansion sont les populations autochtones qui possèdent le droit de planter des espèces pérennes. Les exploitants migrants sont généralement contraints à limiter leurs activités à des cultures annuelles. Cependant, la situation est flexible, particulièrement dans les espaces peu peuplés où les relations de pouvoir et la capacité des migrants riches d'acheter des terres permettent à certains groupes de planter des vergers d'anacardiers sur des surfaces relativement grandes avec l'appui de certaines autorités foncières coutumières. Dans la liste recensant les gagnants et les perdants du boom de l'anacardier au Sud-Ouest du Burkina, les éleveurs transhumants apparaissent comme les principaux perdants. Les jachères qui sont prisées par les éleveurs pour leur grande qualité pastorale étant converties en vergers d'anacardiers, l'accès des transhumants aux pâturages est considérablement réduit. Les conflits entre 
agriculteurs et éleveurs qui sont liés au pâturage des troupeaux dans les vergers non clôturés obligent les transhumants à se déplacer avec leurs troupeaux vers des espaces où les vergers d'anacardiers sont moins présents. En résumé, les processus de territorialisation, qu'ils soient conduits de façon descendante ou ascendante, engendrent des différenciations sociales qui génèrent des possibilités d'enrichissement pour certains et d'appauvrissement pour d'autres.

\section{Conclusion}

Les articles de ce numéro spécial étudient les dynamiques politico-environnementales dans des contextes variés. Les auteurs mettent l'accent sur les processus sociaux et politiques qui produisent de nouvelles configurations socio-spatiales d'accès à la ressource, de son contrôle et de sa gestion. Par contraste avec cette approche sociale relationnelle, les processus écologiques sont peu abordés. Une perspective plus équilibrée pourrait offrir un nouvel éclairage à la conception dominante du territoire qui est celle d'un "espace limité et contrôlable, avec des pourtours démarqués ou des frontières claires, qui sert à faire progresser des objectifs sociaux complexes " (Murphy, 2012, p. 163). Les chercheurs et universitaires qui se sont sérieusement intéressés aux processus écologiques à l'œuvre dans les territoires de conservation et de développement ont observé un décalage spatial entre les limites de tels territoires et les dynamiques écologiques (Turner, 1999 ; Zimmerer, 2000). Ces résultats suggèrent qu'une attention plus grande portée aux relations écologiques, et en particulier à l'influence des facteurs non-humains dans la gestion des ressources naturelles, pourrait conduire à une compréhension plus nuancée des limites du contrôle par la territorialisation.

16 La contribution des articles de ce numéro à la littérature sur la territorialisation est leur analyse sociale relationnelle à la fabrique des territoires de conservation et de développement. La contribution collective est de quatre ordres. Premièrement, les cas d'étude révèlent que les stratégies territoriales émanent d'une grande diversité de localisations et d'aspirations sociales. Les petits agriculteurs familiaux sont tout aussi susceptibles d'engager des pratiques que les agences étatiques. Deuxièmement, les motivations sous-jacentes à la territorialisation sont aussi diverses que les acteurs qui les promeuvent. Étant donné la coalition des acteurs impliqués dans les projets territoriaux, il est peu probable qu'une seule motivation les unisse autour d'un projet territorial. Troisièmement, qui bénéficient finalement d'un projet de territorialisation qui fait débat et où ils réussissent dépend des relations de pouvoir dans des conditions politiques, économiques et géographiques changeantes. Le pouvoir peut être détenu par les autorités de l'État central ou par des alliances territoriales composées d'acteurs situés dans une diversité d'institutions sociales et de localisations géographiques. Enfin, les projets territoriaux décrits dans les cinq cas d'étude révèlent que les acteurs et les institutions sont loin d'être monolithiques. Les usagers des ressources socialement différenciés ne s'expriment pas d'une seule voix, pas plus que les fonctionnaires haut placés dont les intérêts et les alliances varient en fonction de leur engagement institutionnel. Sans surprise, il y a des gagnants et des perdants dans tous les projets territoriaux, ce qui souligne l'importance des approches sociales relationnelles dans l'analyse de la territorialisation. 


\section{BIBLIOGRAPHY}

Bassett T., Blanc-Pamard C., Bourtrais J. 2007. Constructing locality: the terroir approach in West Africa. Africa, 77(1), p. 104-29.

Elden S. 2010. Land, terrain, territory. Progress in Human Geography, 34(6), p. 799-817.

Elden S. 2013. The Birth of Territory. Chicago: University of Chicago Press.

Fairhead J., Leach M., Scoones I., 2012. Green Grabbing: A new appropriation of nature? Journal of Peasant Studies, 39(2), p. 237-261.

Murphy A. 2012. Entente territorial: Sack and Raffestin on territoriality. Environment and Planning D: Society and Space, 30, p. 159-172.

Neumann R., 1998. Imposing Wilderness: Struggles Over Livelihood and Nature Preservation in Africa. University of California Press, Berkeley, CA.

Neumann R. 2004. Nature-state-territory: Toward a critical theorization of conservation enclosures. In R. Peet and M. Watts (eds.), Liberation Ecologies: Environment, development, social movements, ( $2^{\text {nd }}$ edition), New York: Routledge, p. 195-217.

Peluso N., 1993. Coercing Conservation? The politics of state resource control. Global Environmental Change, 3(2), p. 199-217.

Peluso N., Lund C., 2011. New frontiers of land control: Introduction. Journal of Peasant Studies, 38(4), p. 667-681.

Sack R., 1986. Human territoriality. Its theory and history. Cambridge: Cambridge University Press.

Schroeder R., 1999. Geographies of environmental intervention in Africa. PHG, 23(3), p. 359-378.

Scott J., 1998. Seeing Like a State: How Certain Schemes to Improve the Human Condition Have Failed. New Haven, CT, Yale University Press.

Turner M.D., 1999. Conflict, environmental change, and social institutions in dryland Africa: Limitations of the community resource management approach. Society and Natural Resources, 12, p. 643-657.

Wilshusen P.R., Brechin S., Fortwangler C., West P., 2002. Reinventing a squarewheel: critique of a resurgent protection paradigm in international biodiversity conservation. Society and Natural Resources, 15, p. 17-40.

Zimmerer K., 2000. The reworking of conservation geographies: Nonequilibrium landscapes and nature-society hybrids. Annals of the Association of American Geographers, 90(2), p. 356-369.

\section{AUTHORS}

\section{THOMAS J. BASSETT}

Thomas J. Bassett, bassett@illinois.edu, est Professeur de géographie et des SIG, Directeur, LAS Global Studies, University of Illinois à Urbana-Champaign. Il a publié récemment:

- Bassett T., 2014. Capturing the margins: World market prices and cotton farmer incomes in West Africa. World Development, 59, p. 408-421.

- Bassett T., Fogelman C., 2013. Déjà vu or something new? The adaptation concept in the climate 
change literature. Geoforum, 48, p. 42-53.

- Beymer-Farris B., Bassett T., 2012. The REDD Menace: Resurgent protectionism in Tanzania's mangrove forests. Global Environmental Change, 22, p. 332-341.

\section{DENIS GAUTIER}

Denis Gautier, denis.gautier@cirad.fr, est chercheur au CIRAD, département Environnements et Sociétés, Unité de Recherche "Biens et Services des Ecosystèmes Forestiers tropicaux". Il est actuellement seconded scientist au CIFOR (Center for International Forestry Research), basé au Burkina Faso. Il a publié sur cette question :

- Gautier D., 2011. De la gestion centralisée des savanes d'Afrique de l'Ouest aux territoires de conservation et de développement dans un contexte de globalisation environnementale. Dossier d'Habilitation à Diriger des Recherches, Université de Paris 1 Panthéon La Sorbonne, Ecole doctorale de géographie de Paris.

- Gautier D., Benjaminsen (T.A.) (eds.), 2012. Environnement, Discours et Pouvoir. L'approche Political Ecology. Quae, Versailles, $261 \mathrm{p}$.

- Gautier D., Haudidier (B.), Gazull (L.), 2011. Woodcutting and territorial claims in Mali. Geoforum, $42(1)$, p. 28-39. 